\title{
Rough Fuzzy Relation on Two Universal Sets
}

\author{
Xuan Thao Nguyen \\ Faculty of Information Technology, Ha Noi University of Agriculture (HUA), Vietnam \\ E-mail: thaonx281082@yahoo.com \\ Van Dinh Nguyen \\ Faculty of Information Technology, Ha Noi University of Agriculture (HUA), Vietnam \\ E-mail: nvdinh2000@gmail.com \\ Doan Dong Nguyen \\ Faculty of Information Technology, Ha Noi University of Agriculture (HUA), Vietnam \\ E-mail: doandongnguyen@gmail.com
}

\begin{abstract}
Fuzzy set theory was introduced by L.A. Zadeh in 1965. Immediately, it has many applications in practice and in building databases, one of which is the construction of a fuzzy relational database based on similar relationship. The study of cases of fuzzy relations in different environments will help us understand its applications. In this paper, the rough fuzzy relation on Cartesian product of two universe sets is defined, and then the algebraic properties of them, such as the max, min, and composition of two rough fuzzy relations are examined. Finally, reflexive, $\alpha$ reflexive, symmetric and transitive rough fuzzy relations on two universe sets are also defined.
\end{abstract}

Index Terms - Rough Fuzzy Sets, Rough Fuzzy Relations, Similarity Relations

\section{Introduction}

Rough set theory [1] proposed by Pawlak in about 1980 s, is used to handle the redundancies, uncertainties and incorrectness in data mining, as design databases [2] or information systems [3]. This theory has been well developed in both theories and applications. Along with the study of the individual properties of rough set theory, the rough set theory in combination with fuzzy set theory [4] also gains great interest of researchers and becomes a useful tool in exploring the feature selection, the clustering, the control problem, etc. The combination of fuzzy sets and rough sets lead to two concepts [5]: rough fuzzy sets and fuzzy rough sets. Rough fuzzy sets [3, 5, 6, 7] are the fuzzy sets approximated in the crisp approximation spaces and fuzzy rough sets [5] are the crisp sets approximated in the fuzzy approximation spaces. As the construction of fuzzy relations in fuzzy set theory, rough fuzzy relationships building on rough fuzzy set theory has important implications for both theories and applications. In [8], S. Lan has built the fuzzy rough relations on Boolean algebra. By 2011, the authors T. K.
Samanta and B. Sarkar have built the fuzzy rough relations on a given universe set [9]. In this paper, the above results are extended to define the rough fuzzy relations on the Cartesian product of two universe sets, and subsequently their properties are examined.

The remaining parts of this paper is organized as following: In section II, rough fuzzy sets are reintroduced. After studying the rough fuzzy relation and its properties in section III, composition of two rough fuzzy relation and inverse rough fuzzy relation are presented in section IV and section $\mathrm{V}$, respectively. Last but not least, in section VI, the reflexive, symmetric, transitive rough fuzzy relations are studied. Finally, section VII proposes the similarity rough fuzzy relation.

\section{Rough Fuzzy Sets}

Let $U$ be a non-empty set of objects, $R$ is an equivalent relation on $U$. Then the space $(U, R)$ is called an approximation space. Let $X$ be a fuzzy set on $U$. We define the lower approximation set and upper approximation of $X$, respectively

$$
\begin{aligned}
& \underline{a p r}_{R}(X)=\left\{x \in U:[x]_{R} \subset X\right\} \text { where } \\
& \mu_{\underline{\operatorname{apr}_{R}}(X)}=\inf f_{y \in U}\left\{\mu_{X}(y): y \in[x]_{R}\right\}, \\
& \overline{a p r}_{R}(X)=\left\{x \in U:[x]_{R} \cap X \neq \emptyset\right\} \text { where } \\
& \mu_{\overline{a p r}_{R}(X)}=\sup _{y \in U}\left\{\mu_{X}(y): y \in[x]_{R}\right\}, \\
& \text { Boundary of } X, B N D(X)=\overline{a p r}_{R}(X)-\operatorname{apr}_{R}(X) .
\end{aligned}
$$

The fuzzy set $X$ is called a rough fuzzy set if $B N D(X) \neq \emptyset$. 
The Cartesian product of two rough fuzzy sets $X$ and $Y$ is

$$
X \times Y=\{(x, y): x \in U, y \in V\}
$$

where

$$
\mu_{X \times Y}(x, y)=\min \left\{\mu_{X}(x), \mu_{Y}(y)\right\}
$$

\section{Rough Fuzzy Relation and Its Properties}

In this section, we suppose that $U, V$ are two nonempty universal sets and $R_{U_{x}} R_{V}$ are equivalent relations on $U, V$ respectively. Then $R=R_{U} \times R_{V}$ is a equivalent relation on $U \times V$ where

$$
[(u, v)]_{R}=\left\{(x, y) \in U \times V: x \in[u]_{R_{U_{a}}} y \in[v]_{R_{V}}\right\}
$$

Definition 3.1. Let $X, Y$ be the rough fuzzy sets on $U, V$, respectively. We call $\Re \subset U \times V$ is a rough fuzzy relation on $U \times V$ based on the $X \times Y$ if it satisfy

(RFi) $\mu_{\Re}(x, y)=1$, for all $(x, y) \in \underline{X \times Y}$ where $\underline{X \times Y}=\underline{\operatorname{apr}_{R_{U}}}(X) \times \underline{a p r_{R_{V}}}(Y)$.

(RFii) $\mu_{\Re}(x, y)=0$, for all $(x, y) \in U \times V-\overline{X \times Y}$ where $\overline{X \times Y}=\overline{a p r}_{R_{U}}(X) \times \overline{a p r}_{R_{V}}(Y)$.

(RFiii) $0<\mu_{\Re}(x, y)<1$, for all $(x, y) \in \overline{X \times Y}-$ $\underline{X \times Y}$.

Note that:

The conditions (RFi), (RFiii) show that $X \times Y$ is a rough fuzzy set on $U \times V$.

Likewise, the representation of fuzzy relation [10], the rough fuzzy relation can be represented by the graphs, relational tables, matrices.

Definition 3.2. Let $\Re$ is a rough fuzzy relation on $U \times V$ based on the $X \times Y$. For any real numbers $\lambda \in[0,1], R_{\lambda}$ is called $\lambda-$ cut of relation $\Re$ and it was defined following way:

$$
\begin{aligned}
& \Re_{\lambda}(x, y)=1 \Leftrightarrow R(x, y) \geq \lambda \\
& \Re_{\lambda}(x, y)=0 \Leftrightarrow R(x, y)<\lambda
\end{aligned}
$$

Example 3.1. Let $U=\{1,2,3,4\}, V=\{6,7,8,9,10\}$ be two universal sets and $R_{U}=\left\{(x, y): x R_{U} y\right.$ if $x \equiv y(\bmod 2)\}, R_{V}=\left\{(z, t): z R_{V} t\right.$ if $\left.z \equiv t(\bmod 3)\right\}$ are equivalent relations on $U, V$ respectively. $X=\frac{1}{1}+\frac{0.5}{2}+\frac{1}{3}+\frac{0.8}{4}, Y=\frac{0.4}{6}+\frac{0.5}{7}+\frac{1}{8}+\frac{0.8}{9}+\frac{1}{10}$ are rough fuzzy sets on $U, V$ respectively.

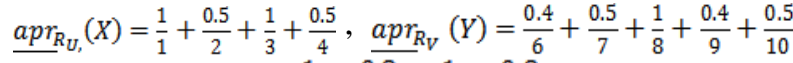
and $\overline{a p r}_{R_{U}}(X)=\frac{1}{1}+\frac{0.8}{2}+\frac{1}{3}+\frac{0.8}{4}, \quad \overline{a p r}_{R_{V}}(Y)=$ $\frac{0.8}{6}+\frac{1}{7}+\frac{1}{8}+\frac{0.8}{9}+\frac{1}{10}$. Here we can define a rough fuzzy relation $\Re$ by a matrix:

$$
M(\Re)=\left[\begin{array}{ccccc}
0 & 0.5 & 1 & 0 & 0.6 \\
0 & 0 & 0 & 0 & 0 \\
0 & 0.5 & 1 & 0 & 0.4 \\
0 & 0 & 0 & 0 & 0
\end{array}\right]
$$

and

$$
\Re_{0.5}=\left[\begin{array}{lllll}
0 & 1 & 1 & 0 & 1 \\
0 & 0 & 0 & 0 & 0 \\
0 & 1 & 1 & 0 & 0 \\
0 & 0 & 0 & 0 & 0
\end{array}\right]
$$

We can see that $\lambda-$ cut of rough fuzzy relation $\Re$ is a crisp relation.

Now we consider some properties of rough fuzzy relations.

Proposition 3.1. Let $\Re_{1}, \Re_{2}$ be two rough fuzzy relations on $U \times V$ based on $X \times Y$. Then $\Re_{1} \wedge \Re_{2}$ where $\quad \mu_{\Re_{1} \wedge \Re_{2}}(x, y)=\min \left\{\mu_{\Re_{1}}(x, y), \mu_{\Re_{2}}(x, y)\right\}$ for all $(x, y) \in U \times V$, is a rough fuzzy set on $U \times V$ based on the $X \times Y$.

\section{Proof.}

We show that $\Re_{1} \wedge \Re_{2}$ satisfy definition 3.1.

(RFi). Since $\mu_{\Re_{1}}(x, y)=\mu_{\Re_{2}}(x, y)=1$ for all $(x, y) \in \underline{X \times Y}$ then $\mu_{\Re_{1} \wedge \Re_{2}}(x, y)=$

$$
\min \left\{\mu_{\Re_{1}}(x, y), \mu_{\Re_{2}}(x, y)\right\}=1 \quad \text { for }
$$
all $(x, y) \in \underline{X \times Y}$.

(RFii). Since $\mu_{\Re_{1}}(x, y)=\mu_{\Re_{2}}(x, y)=0$ for all $(x, y) \in \quad U \times V-\overline{X \times Y} \quad$ then $\mu_{\Re_{1} \wedge \Re_{2}}(x, y)==\min \left\{\mu_{\Re_{1}}(x, y), \mu_{\Re_{2}}(x, y)\right\}=0$ for all $(x, y) \in U \times V-\overline{X \times Y}$.

(RFiii): Since $0<\mu_{\Re_{1}}(x, y), \mu_{\Re_{2}}(x, y)<1$ for all $(x, y) \in \overline{X \times Y}-\underline{X \times Y}$ then $0<\mu_{\Re_{1} \wedge \Re_{2}}(x, y)=$ $\min \left\{\mu_{\Re_{1}}(x, y), \mu_{\Re_{2}}(x, y)\right\}<1 \quad$ for $\quad$ all $(x, y) \in \overline{X \times Y}-\underline{X \times Y}$.

Proposition 3.2. Let $\Re_{1}, \Re_{2}$ be two rough fuzzy relations on $U \times V$ based on $X \times Y$. Then $\Re_{1} \vee \Re_{2}$ where $\quad \mu_{\Re_{1} \vee \Re_{2}}(x, y)=\max \left\{\mu_{\Re_{1}}(x, y), \mu_{\Re_{2}}(x, y)\right\}$ for all $(x, y) \in U \times V$, is a rough fuzzy set on on $U \times V$ based on the $X \times Y$. 


\section{Proof.}

We show that $\Re_{1} \vee \Re_{2}$ satisfy definition 3.1.

(RFi). Since $\mu_{\Re_{1}}(x, y)=\mu_{\Re_{2}}(x, y)=1$ for all $(x, y) \in \underline{X \times Y}$ then $\mu_{\Re_{1} \vee \Re_{2}}(x, y)=$

$$
\max \left\{\mu_{\Re_{1}}(x, y), \mu_{\Re_{2}}(x, y)\right\}=1 \quad \text { for }
$$

$(x, y) \in \underline{X \times Y}$.

(RFii). Since $\mu_{\Re_{1}}(x, y)=\mu_{\Re_{2}}(x, y)=0$ for all $(x, y) \in \quad U \times V-\overline{X \times Y} \quad$ then $\mu_{\Re_{1} \vee \Re_{2}}(x, y)==\max \left\{\mu_{\Re_{1}}(x, y), \mu_{\Re_{2}}(x, y)\right\}=0$ for all $(x, y) \in U \times V-\overline{X \times Y}$.

(RFiii): Since $0<\mu_{\Re_{1}}(x, y), \mu_{\Re_{2}}(x, y)<1$ for all $(x, y) \in \overline{X \times Y}-\underline{X \times Y}$ then $0<\mu_{\Re_{1} \vee \Re_{2}}(x, y)=$ $\max \left\{\mu_{\Re_{1}}(x, y), \mu_{\Re_{2}}(x, y)\right\}<1 \quad$ for $\quad$ all $(x, y) \in \overline{X \times Y}-\underline{X \times Y}$. $\square$

Lemma 3.1. If $0<a, b<1$ then

i) $0<a b<1$ (obvious)

ii) $0<a+b-a b<1$

Indeed, since $0<a, b<1 \quad$ then $a+b \geq 2 \sqrt{a b}>2 a b>a b>0, \quad$ therefore $a+b-a b>0$. On the other hand $1-(a+b-a b)=(1-a)(1-b)>0 \quad$ then $a+b-a b<1$.

The following properties of rough fuzzy relations are obtained by using these algebraic results:

Proposition 3.3. Let $\Re_{1}, \Re_{2}$ be two rough fuzzy relations on $U \times V$ based on $X \times Y$. Then $\Re_{1} \otimes \Re_{2}$ where $\mu_{\Re_{1}} \otimes \Re_{2}(x, y)=\mu_{\Re_{1}}(x, y) \cdot \mu_{\Re_{2}}(x, y)$ for all $(x, y) \in U \times V$, is a rough fuzzy set on on $U \times V$ based on the $X \times Y$.

\section{Proof.}

The relation $\Re_{1} \otimes \Re_{2}$ is satisfied definition 3.1. Indeed:

(RFi). Since $\mu_{\Re_{1}}(x, y)=\mu_{\Re_{2}}(x, y)=1$ for all $(x, y) \in \underline{X \times Y}$ then $\mu_{\Re_{1} \otimes \Re_{2}}(x, y)=$

$$
\mu_{\Re_{1}}(x, y) \cdot \mu_{\Re_{2}}(x, y)=1 \text { for all }(x, y) \in \underline{X \times Y} .
$$

(RFii). Since $\mu_{\Re_{1}}(x, y)=\mu_{\Re_{2}}(x, y)=0$ for all $(x, y) \in \quad U \times V-\overline{X \times Y} \quad$ then $\mu_{\Re_{1} @ \Re_{2}}(x, y)=\mu_{\Re_{1}}(x, y) \cdot \mu_{\Re_{2}}(x, y)=0$ for all $(x, y) \in U \times V-\overline{X \times Y}$.
(RFiii): Since $0<\mu_{\Re_{1}}(x, y), \mu_{\Re_{2}}(x, y)<1$ for all $(x, y) \in \overline{X \times Y}-\quad \underline{X \times Y} \quad$ then $0<\mu_{\Re_{1} \otimes \Re_{2}}(x, y)=\mu_{\Re_{1}}(x, y) \cdot \overline{\mu_{\Re_{2}}(x, y)<1}$ for all $(x, y) \in \overline{X \times Y}-\underline{X \times Y}$ (Lemma $3.1 \mathrm{i})$ ).

Proposition 3.4. Let $\Re_{1}, \Re_{2}$ be two rough fuzzy relations on $U \times V$ based on $X \times Y$. Then $\Re_{1} \oplus \Re_{2}$ where

$\mu_{\Re_{1} \oplus \Re_{2}}(x, y)=\mu_{\Re_{1}}(x, y)+\mu_{\Re_{2}}(x, y)-$ $\mu_{\Re_{1}}(x, y) \cdot \mu_{\Re_{2}}(x, y)$

for all $(x, y) \in U \times V$, is a rough fuzzy set on $U \times V$ based on the $X \times Y$.

\section{Proof.}

We show that $\Re_{1} \oplus \Re_{2}$ satisfy definition 3.1.

(RFi). Since $\mu_{\Re_{1}}(x, y)=\mu_{\Re_{2}}(x, y)=1$ for all $(x, y) \in \underline{X \times Y}$ then $\mu_{\Re_{1} \oplus \Re_{2}}(x, y)=$

$\mu_{\Re_{1}}(x, y)+\mu_{\Re_{2}}(x, y)-\mu_{\Re_{1}}(x, y) \cdot \mu_{\Re_{2}}(x, y)=1$ for all $(x, y) \in \underline{X \times Y}$.

(RFii). Since $\mu_{\Re_{1}}(x, y)=\mu_{\Re_{2}}(x, y)=0$ for all $(x, y) \in \quad U \times V-\overline{X \times Y} \quad$ then $\mu_{\Re_{1} \oplus \Re_{2}}(x, y)=\mu_{\Re_{1}}(x, y)+\mu_{\Re_{2}}(x, y)-$ $\mu_{\Re_{1}}(x, y) \cdot \mu_{\Re_{2}}(x, y)=0$ for all $(x, y) \in U \times V-\overline{X \times Y}$.

(RFiii): Since $0<\mu_{\Re_{1}}(x, y), \mu_{\Re_{2}}(x, y)<1$ for all $(x, y) \in \overline{X \times Y}-\quad X \times Y \quad$ then $0<\mu_{\Re_{1} \oplus \Re_{2}}(x, y)=\mu_{\Re_{1}}(x, y)+\overline{\mu_{\Re_{2}}}(x, y)-$ $\mu_{\Re_{1}}(x, y) \cdot \mu_{\Re_{2}}(x, y)<1$ for all $(x, y) \in \overline{X \times Y}-\underline{X \times Y}$ (Lemma 3.1 ii)).

\section{Composition of Two Rough Fuzzy Relations}

Let $U, V, W$ be the universal sets. $\Re_{1}, \Re_{2}$ are two rough fuzzy relations on $U \times V, V \times W$ based on $X \times Y, Y \times Z$, respectively.

Definition 4.1. Composition of two rough fuzzy relations $\Re_{1}, \Re_{2}$ denote $\Re_{1} \circ \Re_{2}$ which defined on $U \times W$ based on $X \times Z$ where

$$
\begin{aligned}
& \mu_{\Re_{1} \circ \Re_{2}}(x, z)=\max _{y \in V}\left\{\min \left[\mu_{\Re_{1}}(x, y), \mu_{\Re_{2}}(y, z)\right]\right\} \\
& \text { for all }(x, z) \in U \times W .
\end{aligned}
$$

Proposition 4.1. $\Re_{1} \circ \Re_{2}$ is a rough fuzzy relation $\mathrm{R}$ on $U \times W$ based on $X \times Z$.

Proof. 
(RFi) Since $\Re_{1}, \Re_{2}$ are two rough fuzzy relations on $U \times V, V \times W$ based on $X \times Y, Y \times Z$, respectively. Then $\mu_{\Re_{1}}(x, y), \mu_{\Re_{2}}(y, z)=1$ for all $(x, y) \in \underline{X \times Y}$ and $(y, z) \in \underline{Y \times Z}$. We denote $\underline{Y}=\underline{a p r_{R_{V}}}(Y)$ and we have

$$
\begin{aligned}
& \mu_{\Re_{1} \circ \Re_{2}}(x, z) \\
& =\max _{y \in V}\left\{\min _{(x ; z) \in X \times Z}\left[\mu_{\Re_{1}}(x, y), \mu_{\Re_{2}}(y, z)\right]\right\} \\
& =\max _{y \in \underline{Y}}\left\{\min _{(x, z) \in \underline{X \times Z}}\left[\mu_{\Re_{1}}(x, y), \mu_{\Re_{2}}(y, z)\right]\right\} \\
& \vee \max _{v \in V-\underline{Y}}\left\{\min _{(x, z) \in \underline{X \times Z} \underline{\Re_{1}}}\left[\mu_{\Re_{1}}(x, v), \mu_{\Re_{2}}(v, z)\right]\right\} \\
& = \\
& \checkmark \max _{v \in V-\underline{Y}}\left\{\min _{(x, z) \in \underline{X \times Z}}\left[\mu_{\Re_{1}}(x, v), \mu_{\Re_{2}}(v, z)\right]\right\} \\
& =1 \text { for all }(x, z) \in \underline{X \times Z} \text {. }
\end{aligned}
$$

(RFii) Note that $\mu_{\Re_{1}}(x, y), \mu_{\Re_{2}}(y, z)=0$ for all $(x, y) \in U \times V-\overline{X \times Y}$ and $(y, z) \in V \times W-\overline{Y \times Z}$.

We consider $\mu_{\Re_{1} \circ \Re_{2}}(x, z)$

$=\max _{y \in V}\left\{\min _{(x, z) \in U \times W-\overline{X \times Z}}\left[\mu_{\Re_{1}}(x, y), \mu_{\Re_{2}}(y, z)\right]\right\}$

For all $(x, z) \in U \times W-\overline{X \times Z}$, it exists $x \notin \overline{\operatorname{apr}}_{R}(X) \quad$ so $\quad(x, v) \in U \times V-\overline{X \times Y} \quad$ and $\mu_{\Re_{1}}(x, v)=0$ for all $v \in V$. Similarly, it exists $z \notin \overline{\operatorname{apr}}_{R}(Z) \quad$ so $\quad(v, z) \in V \times W-\overline{Y \times Z} \quad$ and $\mu_{\Re_{2}}(v, z)=0$ for all $v \in V$.

Hence $\min _{(x, z) \in X \times Z}\left[\mu_{\Re_{1}}(x, v), \mu_{\Re_{2}}(v, z)\right]=0$ for all $(x, z) \in U \times W-\overline{X \times Z}$ and $v \in V$. So that $\mu_{\Re_{1} \circ \Re_{2}}(x, z)$

$=\max _{y \in V}\left\{\min _{(x, z) \in U \times W-\overline{X \times Z}}\left[\mu_{\Re_{1}}(x, y), \mu_{\Re_{2}}(y, z)\right]\right\}$ $=0$ for all $(x, z) \in U \times W-\overline{X \times Z}$.

(RFiii) We must proof

$0<\max _{y \in V}\left\{\min \left[\mu_{\Re_{1}}(x, y), \mu_{\Re_{2}}(y, z)\right]\right\}<1 \quad$ for all $(x, z) \in \overline{X \times Z}-\underline{X \times Z}$.

Since $(x, z) \notin \underline{X \times Z}$ then at least existing $x \notin \underline{X}={\underline{a p r_{R_{U}}}}(X)$ or $z \notin \underline{Z}={\underline{a p r_{R_{W}}}}(Z)$ so that $\min \left[\mu_{\Re_{1}}(x, y), \mu_{\Re_{2}}(y, z)\right]<1$ for all $y \in Y$. On the other hand, $\mu_{\Re_{1}}(x, v)=\mu_{\Re_{2}}(v, z)=0$ for all $(x, z) \in U \times W-\overline{X \times Z}$ and $v \in V$ then we have $0<\left\{\min \left[\mu_{\Re_{1}}(x, y), \mu_{\Re_{2}}(y, z)\right]\right\}<1 \quad$ for $\quad$ all $(x, z) \in \overline{X \times Z}-\underline{X \times Z}$. and $y \in Y$. Hence, we have $0<\max _{y \in V}\left\{\min \left[\mu_{\Re_{1}}(x, y), \mu_{\Re_{2}}(y, z)\right]\right\}<1 \quad$ or $0<\mu_{\Re_{1} \circ \Re_{2}}(x, z)<1$ for all $(x, z) \in \overline{X \times Z}-\underline{X \times Z}$.

Proposition 4.2. Let $U, V, W, W^{\prime}$ be the universe sets. $\Re_{1}, \Re_{2}, \Re_{3}$ are rough fuzzy relations on
$U \times V, V \times W, W \times W^{\prime}$ based on $X \times Y, Y \times Z, Z \times Z^{\prime}$, respectively. Then $\left(\Re_{1} \circ \Re_{2}\right) \circ \Re_{3}=$ $\Re_{1} \circ\left(\Re_{2} \circ \Re_{3}\right)$

Proof.

For all $x \in U, y \in V, z \in W, t \in W^{\prime}$ we have

$$
\begin{aligned}
& \quad \mu_{\left.\Re_{1} \circ \Re_{2} \circ \Re_{3}\right)}(x, t) \\
& =\max _{y \in V}\left\{\min \left[\mu_{\Re_{1}}(x, y), \mu_{\Re_{2} \circ \Re_{3}}(y, t)\right]\right\} \\
& = \\
& \max _{y \in V}\left\{\operatorname { m i n } \left\{\mu_{\Re_{1}}(x, y), \max _{z \in W}\left\{\operatorname { m i n } \left[\mu_{\Re_{2}}(y, z),\right.\right.\right.\right. \\
& \left.\left.\left.\left.\mu_{\Re_{3}}(z, t)\right]\right\}\right\}\right\} \\
& = \\
& \max _{z \in W}\left\{\operatorname { m i n } \left\{\operatorname { m a x } _ { y \in V } \left\{\min \left[\mu_{\Re_{1}}(x, y), \mu_{\Re_{2}}(y, z)\right],\right.\right.\right. \\
& \left.\left.\left.\left.\mu_{\Re_{3}}(z, t)\right]\right\}\right\}\right\} \\
& =\max _{z \in W}\left\{\min \left[\mu_{\Re_{1} \circ \Re_{2}}(x, y), \mu_{\Re_{3}}(y, t)\right]\right\} \\
& =\mu_{\left(\Re_{1} \circ \Re_{2}\right) \circ \Re_{3}}(x, t) . \square
\end{aligned}
$$

We note that $\Re_{1} \circ \Re_{2} \neq \Re_{2} \circ \Re_{1}$, because the composition of two rough fuzzy relations $\Re_{1}, \Re_{2}$ exists but the composition of two rough fuzzy relations $\Re_{2}, \Re_{1}$ does not exist necessarily.

\section{Inverse Rough Fuzzy Relation}

Let $X$ and $Y$ be the two rough fuzzy sets on $U$ and $V$, respectively. $\Re \subset U \times V$ is a rough fuzzy relation on $U \times V$ based on $X \times Y$. Then we define $\Re^{-1} \subset V \times U$ is a rough fuzzy relation on $V \times U$ based on $Y \times X$ as following:

$$
\mu_{\Re^{-1}}(y, x)=\mu_{\Re}(x, y) \text { for all }(y, x) \in V \times U .
$$

Definition 5.1. The relation $\Re^{-1}$ is called the inverse rough fuzzy relation of $\Re$.

\section{Proposition 5.1.}

i) $\left(\Re^{-1}\right)^{-1}=\Re$.

ii) Let $\Re_{1}, \Re_{2}$ be two rough fuzzy relations on $U \times V, V \times W$ based on $X \times Y, Y \times Z$, respectively. Then $\left(\Re_{1} \circ \Re_{2}\right)^{-1}=\Re_{2}^{-1} \circ \Re_{1}^{-1}$.

Proof.

i) $\mu_{\left.\mathscr{R}^{-1}\right)^{-1}}(x, y)=\mu_{\Re^{-1}}(y, x)=\mu_{\Re}(x, y)=1$, for all $(x, y) \in \underline{X \times Y}$

$$
\mu_{\left(\Re^{-1}\right)^{-1}}(x, y)=\mu_{\Re^{-1}}(y, x)=\mu_{\Re}(x, y)=0 \text {, for all }
$$
$(x, y) \in U \times V-\overline{X \times Y}$

I.J. Intelligent Systems and Applications, 2014, 04, 49-55 
$0<\mu_{\left(\Re^{-1}\right)^{-1}}(x, y)=\mu_{\Re-1}(y, x)=\mu_{\Re}(x, y)<1$ for all $(x, y) \in \overline{X \times Y}-\underline{X \times Y}$.

It means $\left(\Re^{-1}\right)^{-1}=\Re$.

ii) For all $x \in U, y \in V, z \in W$ we have

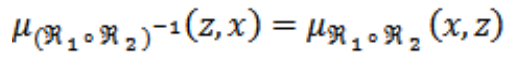

$$
\begin{aligned}
& =\max _{y \in V}\left\{\min \left[\mu_{\Re_{1}}(x, y), \mu_{\Re_{2}}(y, z)\right]\right\} \\
& =\max _{y \in V}\left\{\min \left[\mu_{\Re_{1}}(x, y), \mu_{\Re_{2}}(y, z)\right]\right\} \\
& =\max _{y \in V}\left\{\min \left[\mu_{\Re_{2}}{ }^{-2}(z, y), \mu_{\Re_{1}}{ }^{-1}(y, x)\right]\right\} \\
& =\mu_{\Re_{2}-1 \Re_{1}}{ }^{-1}(z, x)
\end{aligned}
$$

That means $\left(\Re_{1} \circ \Re_{2}\right)^{-1}=\Re_{2}{ }^{-1} \circ \Re_{1}^{-1}$. $\square$

In the same way, the representation of fuzzy relation, we can represent the rough fuzzy relation $\Re$ by using matrix $M(\Re)$. So that, the inverse rough fuzzy relation $\Re^{-1}$ of rough fuzzy relation $\Re$ by using matrix $M(\Re)^{t}$, it is the transposition of the matrix $M(\Re)$.

Example 5.1. Let $U=\{1,2,3,4\}, V=\{6,7,8,9,10\}$ be two universal sets and $R_{U}=\left\{(x, y): x R_{U} y\right.$ if only if $x \equiv y(\bmod 2)\}, R_{V}=\left\{(z, t): z R_{V} t\right.$ if $\left.z \equiv t(\bmod 3)\right\}$ are equivalent relations on $U, V$ respectively. $X=\frac{1}{1}+\frac{0.5}{2}+\frac{1}{3}+\frac{0.8}{4}, Y=\frac{0.4}{6}+\frac{0.5}{7}+\frac{1}{8}+\frac{0.8}{9}+\frac{1}{10}$ are rough fuzzy sets on $U, V$, respectively. ${\underline{a p r_{R_{U}}}}_{(X)}(X) \frac{1}{1}+\frac{0.5}{2}+\frac{1}{3}+\frac{0.5}{4}, \underline{a p r}_{R_{V}}(Y)=\frac{0.4}{6}+\frac{0.5}{7}+\frac{1}{8}+\frac{0.4}{9}+\frac{0.5}{10}$ and $\overline{a p r}_{R_{U}}(X)=\frac{1}{1}+\frac{0.8}{2}+\frac{1}{3}+\frac{0.8}{4}, \quad \overline{a p r}_{R_{V}}(Y)=$ $\frac{0.8}{6}+\frac{1}{7}+\frac{1}{8}+\frac{0.8}{9}+\frac{1}{10}$. Here we can define a rough fuzzy relation $\Re$ as following:

$$
M(\Re)=\left[\begin{array}{ccccc}
0 & 0.5 & 1 & 0 & 0.6 \\
0 & 0 & 0 & 0 & 0 \\
0 & 0.5 & 1 & 0 & 0.4 \\
0 & 0 & 0 & 0 & 0
\end{array}\right]
$$

Then $\Re^{-1}$ is an inverse rough fuzzy relation of $\Re$, it is represented by using matrix

$$
M\left(\Re^{-1}\right)=M(\Re)^{t}=\left[\begin{array}{llcl}
0 & 0 & 0 & 0 \\
0.5 & 0 & 0.5 & 0 \\
1 & 0 & 1 & 0 \\
0 & 0 & 0 & 0 \\
0.6 & 0 & 0.4 & 0
\end{array}\right]
$$

\section{The Reflexive, Symmetric, Transitive Rough Fuzzy Relation}

In this section, we consider some properties of rough fuzzy relation on a set, such as reflexive, symmetric, transitive properties.

Copyright @ 2014 MECS
Let $(U, R)$ be a crisp approximation space and $X$ is a rough fuzzy set on $(U, R)$. We consider rough fuzzy relation in definition 3.1 in the case $U \equiv V, R_{U} \equiv R_{V} \equiv R$, and $X \equiv Y$. From here onwards, the rough fuzzy relation $\Re$ is called rough fuzzy relation on $(U, R)$ based on the rough fuzzy set $X$.

Definition 6.1. The rough fuzzy relation $\Re$ is said to be reflexive rough fuzzy relation if $\mu_{\Re}(x, x)=1$ for all $(x, x) \in U \times U, \mu_{X}(x)>0$.

Proposition 6.1. Let $\Re_{1}, \Re_{2}$ be two rough fuzzy relation on $U$ based $X$. If $\Re_{1}, \Re_{2}$ are the reflexive rough fuzzy relations then $\Re_{1} \wedge \Re_{2}$, $\Re_{1} \vee \Re_{2}, \Re_{1} \otimes \Re_{2}, \Re_{1} \oplus \Re_{2}, \Re_{1} \circ \Re_{2}$, also.

\section{Proof.}

If $\Re_{1}, \Re_{2}$ are the reflexive rough fuzzy relations then $\mu_{\Re_{1}}(x, x), \mu_{\Re_{2}}(x, x)=1$ for all $(x, x) \in U \times U$, $\mu_{X}(x)>0$. We have

$$
\mu_{\Re_{1} \wedge \Re_{2}}(x, x)=\min \left\{\mu_{\Re_{1}}(x, x), \mu_{\Re_{2}}(x, x)\right\}=1
$$

for all $(x, x) \in U \times U, \quad \mu_{X}(x)>0$ and $\Re_{1} \wedge \Re_{2}$ is reflexive rough fuzzy relation.

$$
\mu_{\Re_{1} \vee \Re_{2}}(x, x)=\max \left\{\mu_{\Re_{1}}(x, x), \mu_{\Re_{2}}(x, x)\right\}=1
$$

for all $(x, x) \in U \times U, \quad \mu_{X}(x)>0 \quad$ and $\Re_{1} \vee \Re_{2}$ reflexive rough fuzzy relation.

$\mu_{\Re_{1} \otimes \Re_{2}}(x, x)=\mu_{\Re_{1}}(x, x) \cdot \mu_{\Re_{2}}(x, x)=1$ for all $(x, x) \in U \times U, \quad \mu_{X}(x)>0$ and $\Re_{1} \otimes \Re_{2}$ reflexive rough fuzzy relation.

$$
\begin{aligned}
& \mu_{\Re_{1} \oplus \Re_{2}}(x, x)=\mu_{\Re_{1}}(x, x)+\mu_{\Re_{2}}(x, x)- \\
& \mu_{\Re_{1}}(x, x) \cdot \mu_{\Re_{2}}(x, x)=1
\end{aligned}
$$

for all $(x, x) \in U \times U, \quad \mu_{X}(x)>0 \quad$ and $\Re_{1} \oplus \Re_{2}$ reflexive rough fuzzy relation.

$$
\begin{aligned}
& \quad \mu_{\Re_{1} \circ \Re_{2}}(x, x)= \\
& \quad \max _{y \in U}\left\{\min \left[\mu_{\Re_{1}}(x, y), \mu_{\Re_{2}}(y, x)\right]\right\}=1 \\
& \text { for all } \quad(x, x) \in U \times U, \quad \mu_{X}(x)>0 \quad \text { and } \\
& \Re_{1} \circ \Re_{2} \text { reflexive rough fuzzy relation. } \square
\end{aligned}
$$

Definition 6.2. The rough fuzzy relation $\Re$ is said to be $\alpha$-reflexive rough fuzzy relation where $\propto=\min _{\left[x_{i}\right]_{R} \subset U} \propto_{i}$ if $\mu_{\Re}\left(x_{i}, y\right)=\propto_{i}$ for all $\left(x_{i}, y\right) \in U \times U, y \in\left[x_{i}\right]_{R}$ and $\mu_{X}(x)>0$.

Proposition 6.2. Let $\Re_{1}, \Re_{2}$ be two rough fuzzy relation on $U$ based $X$. If $\Re_{1}, \Re_{2}$ are the $\alpha$-reflexive rough fuzzy relations then $\Re_{1} \wedge \Re_{2}, \Re_{1} \vee \Re_{2}$ also.

\section{Proof.}

If $\Re_{1}, \Re_{2}$ are the $\alpha$-reflexive rough fuzzy relations then $\quad \mu_{\Re_{1}}\left(x_{i}, y\right)=\mu_{\Re_{2}}\left(x_{i}, y\right)=\propto \quad$ where 
$\propto=\min _{\left[x_{i}\right]_{R} \subset U} \propto_{i}$ if $\quad \mu_{\Re_{1}}\left(x_{i}, y\right)=\mu_{\Re_{2}}\left(x_{i}, y\right)=\propto_{i}$ for all $\left(x_{i}, y\right) \in U \times U, y \in\left[x_{i}\right]_{R}$ and $\mu_{X}(x)>0$. We have

$$
\mu_{\Re_{1} \wedge \Re_{2}}\left(x_{i}, y\right)=\min \left\{\mu_{\Re_{1}}\left(x_{i}, y\right), \mu_{\Re_{2}}\left(x_{i}, y\right)\right\}=\propto
$$

where

$$
\propto=\min _{\left[x_{i}\right]_{R} \subset U} \propto_{i}
$$

$\mu_{\Re_{1}}\left(x_{i}, y\right)=\mu_{\Re_{2}}\left(x_{i}, y\right)=\propto_{i}$ for all $\left(x_{i}, y\right) \in U \times U$, $y \in\left[x_{i}\right]_{R}$ and $\mu_{X}(x)>0$ for all $(x, x) \in U \times U$, $\mu_{X}(x)>0$ and $\Re_{1} \wedge \Re_{2} \alpha$-reflexive rough fuzzy relation.

$$
\begin{aligned}
& \mu_{\Re_{1} \vee \Re_{2}}\left(x_{i}, y\right)=\max \left\{\mu_{\Re_{1}}\left(x_{i}, y\right), \mu_{\Re_{2}}\left(x_{i}, y\right)\right\}=\propto \\
& \text { where } \quad \propto=\min _{\left[x_{i}\right]_{R} \subset U} \propto_{i} \quad \text { if }
\end{aligned}
$$
$\mu_{\Re_{1}}\left(x_{i}, y\right)=\mu_{\Re_{2}}\left(x_{i}, y\right)=\propto_{i}$ for all $\left(x_{i}, y\right) \in U \times U$, $y \in\left[x_{i}\right]_{R}$ and $\mu_{X}(x)>0$ for all $(x, x) \in U \times U$, $\mu_{X}(x)>0$ and $\Re_{1} \vee \Re_{2} \alpha$-reflexive rough fuzzy relation.

Definition 6.3. The rough fuzzy relation $\Re$ is said to be symmetric rough fuzzy relation if $\mu_{\Re}(x, y)=\mu_{\Re}(y, x)$ for all $(x, y) \in U \times U$.

We note that if $\Re$ is a symmetric rough fuzzy relation then matrix $M(\Re)$ is a symmetric matrix.

It is easy to see that:

Proposition 6.3. Let $\Re_{1}, \Re_{2}$ be two rough fuzzy relations on $U$ based rough fuzzy set. If $\Re_{1}, \Re_{2}$ are the symmetric rough fuzzy relations then $\Re_{1} \wedge \Re_{2}$, $\Re_{1} \vee \Re_{2}, \Re_{1} \otimes \Re_{2}, \Re_{1} \oplus \Re_{2}, \Re_{1} \circ \Re_{2}$ also.

Definition 6.4. The rough fuzzy relation $\Re$ is said to be transitive rough fuzzy relation if $\Re \circ \Re \subseteq \Re$.

\section{Similarity Rough Fuzzy Relation}

Definition 7.1. The rough fuzzy relation $\Re$ on $U$ based on the rough fuzzy set $X$ is called a similarity rough fuzzy relation if it has the reflexive, symmetric, transitive.

Definition 7.2. The rough fuzzy relation $\Re$ on $U$ based on the rough fuzzy set $X$ is called a $\alpha$ - similarity rough fuzzy relation if it has the $\alpha$-reflexive, symmetric, transitive.

Now, we consider an illustration example.

Example 7.1. We consider the decision system in Table 1. In which $\{A, B, C\}$ is the collection of condition attributes and $D$ is the decision attribute.
Table 1: A decision system

\begin{tabular}{|c|c|c|c|c|}
\hline At U & A & B & C & D \\
\hline $\mathrm{u} 1$ & 0.1 & 0.2 & 0.3 & Y \\
\hline $\mathrm{u} 2$ & 0.1 & 0.3 & 0.4 & N \\
\hline $\mathrm{u} 3$ & 0.2 & 0.3 & 0.5 & $\mathrm{Y}$ \\
\hline $\mathrm{u} 4$ & 0.2 & 0.3 & 0.5 & $\mathrm{Y}$ \\
\hline $\mathrm{u} 5$ & 0.6 & 0.4 & 0.3 & $\mathrm{~N}$ \\
\hline
\end{tabular}

$R=I N D(A, B, C)$ is an equivalent relation on $U$, $(U, R)$ is a crisp approximation space and $U / R=\{\{u 1\},\{u 2\},\{u 3, u 4\},\{u 5\}\}$.

We consider $F=\frac{0.1}{u 1}+\frac{0.2}{u 2}+\frac{0.4}{u 3}+\frac{1}{u 4}+\frac{1}{u 5}$ is a fuzzy set in $U$. We compute

$$
\begin{aligned}
& \underline{\operatorname{apr}_{R}}(F)=\frac{0.1}{u 1}+\frac{0.2}{u 2}+\frac{0.4}{u 3}+\frac{0.4}{u 4}+\frac{1}{u 5} \\
& \overline{\operatorname{apr}}_{R}(F)=\frac{0.1}{u 1}+\frac{0.2}{u 2}+\frac{1}{u 3}+\frac{1}{u 4}+\frac{1}{u 5} .
\end{aligned}
$$

It is easy that $\overline{a p r}_{R}(F) \neq \operatorname{apr}_{R}(F)$, so $F$ is a rough fuzzy set on $U$.

We can put $\mu_{\Re_{F}}(u 5, u 5)=1 ; \mu_{\Re_{F}}(u i, u j)=1$, if $i, j=1,2$ and $0<\mu_{\Re_{F}}(u i, u j)<1$ in the other cases.

$$
M\left(\Re_{F}\right)=\left[\begin{array}{ccccc}
0 & 0 & r_{13} & r_{14} & r_{15} \\
0 & 0 & r_{23} & r_{24} & r_{25} \\
r_{31} & r_{32} & r_{33} & r_{34} & r_{35} \\
r_{41} & r_{42} & r_{43} & r_{44} & r_{45} \\
r_{51} & r_{52} & r_{53} & r_{54} & 1
\end{array}\right]
$$

According the definition 3.1 we have $\Re_{F}$ is a rough fuzzy relation on $U$ based on $F$.

Note that, the fuzzy set $F_{1}=\frac{0.1}{u 1}+\frac{0.2}{u 2}+\frac{0.4}{u 3}+\frac{0.4}{u 4}+\frac{1}{u 5}$ is not the rough fuzzy set on $U$ because

$$
\begin{aligned}
& \operatorname{apr}_{R}\left(F_{1}\right)=\frac{0.1}{u 1}+\frac{0.2}{u 2}+\frac{0.4}{u 3}+\frac{0.4}{u 4}+\frac{1}{u 5} \\
& =\overline{a p r}_{R}\left(F_{1}\right)=\frac{0.1}{u 1}+\frac{0.2}{u 2}+\frac{0.4}{u 3}+\frac{0.4}{u 4}+\frac{1}{u 5} .
\end{aligned}
$$

We consider $F_{2}=\frac{1}{u 1}+\frac{1}{u 2}+\frac{0.4}{u 3}+\frac{1}{u 4}+\frac{1}{u 5}$ is a rough fuzzy set on $U$ with

$$
\begin{aligned}
& \underline{F_{2}}=\underline{a p r_{R}}\left(F_{2}\right)=\frac{1}{u 1}+\frac{1}{u 2}+\frac{0.4}{u 3}+\frac{1}{u 4}+\frac{1}{u 5} \text { and } \\
& \bar{F}_{2}=\overline{a p r}_{R}\left(F_{2}\right)=\frac{1}{u 1}+\frac{1}{u 2}+\frac{1}{u 3}+\frac{1}{u 4}+\frac{1}{u 5}=U .
\end{aligned}
$$

A rough fuzzy relation on $U$ based on $F_{2}$ where 


$$
M\left(\Re_{F_{2}}\right)=\left[\begin{array}{ccccc}
1 & 1 & 0 & 0 & 1 \\
1 & 1 & 0 & 0 & 1 \\
0 & 0 & \frac{1}{2} & \frac{1}{2} & 0 \\
0 & 0 & \frac{1}{2} & \frac{1}{2} & 0 \\
1 & 1 & 0 & 0 & 1
\end{array}\right] .
$$

$\Re_{F_{2}}$ is clearly the $\frac{1}{2}$ - reflexive, symmetric rough fuzzy relation.

$$
\text { We have } M\left(\Re_{F_{2}} \circ{ }^{\circ} F_{2}\right)=\left[\begin{array}{ccccc}
1 & 1 & 0 & 0 & 1 \\
1 & 1 & 0 & 0 & 1 \\
0 & 0 & \frac{1}{2} & \frac{1}{2} & 0 \\
0 & 0 & \frac{1}{2} & \frac{1}{2} & 0 \\
1 & 1 & 0 & 0 & 1
\end{array}\right] \text {. }
$$

It is obvious that $M\left(\Re_{E_{2}} \circ \Re_{F_{2}}\right) \leq M\left(\Re_{E_{2}}\right)$ then $\Re_{F_{2}}$ is the transitive rough fuzzy relation; so that, $\Re_{F_{2}}$ is a $\frac{1}{2}$ - similarity relation.

\section{Conclusion}

In this paper, we have developed the rough fuzzy relation on two universe sets and studied some theoretical results of rough fuzzy relations. In the future, we will explore the information measures based on rough fuzzy relations and apply in the feature selection problems, as well as find the attribute reduction sets in the data table or build databases based on rough fuzzy relations.

\section{References}

[1] Z. Pawlak, Rough sets, International Journal of computer and information sciences 11(5), 341 $356,1982$.

[2] T. Beaubouef, F. Petry, Normalization in rough relation database, International conference on rough sets, fuzzy sets, data mining and granular computing, pp 257-265, 2005.

[3] T. Beaubouef, F. Petry, Rough and rough fuzzy sets in design of information systems, Computational Complexity: theory, technique and application, pp 2702-2715, 2012.

[4] L.A. Zadeh, Fuzzy set, Information and control (8), pp 338-353, 1965.

[5] Y.Y.Yao, Combination of rough and fuzzy sets based on $\alpha$ - level sets, Rough sets and Data mining: analysis for imprecise data, Kluwer Academic Publisher, Boston, pp 301 - 321, 1997.

[6] A. Skowron, Rough - fuzzy computing, Handbook of natural computing, Springer - Verlarg Berlin Heidelberg, pp 1921 - 1948, 2012.

[7] W. Z. Wu, Y. H. Xu, On fuzzy topological structures of rough fuzzy sets, Transactions on rough sets XVI, LNCS 7736, Springer - Verlag Berlin Heidelberg, pp 125-143, 2013.

[8] S. Lan, Fuzzy rough relation and Its properties, Journal of Systems science and Systems Engineering, V. 11, N. 3, pp 367 - 370, 2002.

[9] T. K. Samanta, B. Sarkar, Fuzzy rough relation, G.J.P and A Sc and Tech, Vol 01, pp 10 - 17, 2011.

[10] W. B. V. Kandasamy and F. Smarandache, Fuzzy relational maps and neutrosophic relational maps, HEXIS Church Rock, 2004.

\section{Authors' Profile}

X.T. Nguyen was born on October 28, 1982, in Thai Binh, Viet Nam. He received the B.Sc. and M.S Degrees, in Mathematic from The College of Science Vietnamese National University (VNU), Hanoi, in 2004 and 2009, respectively. Now, he is a lecturer, Department of applied Math-Informatics, Faculty of Information Technology, Hanoi University of Agriculture (HUA), Viet Nam. He is teaching Calculus, Optimization, Fuzzy logic and application. His research interests are Spectral theory of operator Fuzzy set theory, Rough set theory and application in data mining. His representative published article: Remarks on the spectrum of a compact convex set of compact operators, Journal of Applied Analysis 16 (2010), pp 259-264.

V.D. Nguyen was born on the year 1953 in Hanoi, Vietnam. He was a post-graduate intern in computer Science in Moscow, The Russian Federation. In 2004 he received the Doctor of Philosophy $(\mathrm{PhD})$ Degree in Mathematics at the College of Science, Vietnam National University (VNU), Hanoi. Now he is an Associate Professor in the Department of applied MathInformatics, Faculty of Information Technology, Hanoi University of Agriculture (HUA).

D.D. Nguyen, born on the year 1986, in Haiduong, Vietnam, had B.Sc. in Electronic and telecommunication in 2009 and M.Sc. in Electronic in 2011 at Hanoi University of Science and Technology. Since 2010, he has been working as a lecturer at Software Engineering Department, Faculty of Information Technology, Hanoi University of Agriculture. He has done a project: "Studying and Implementation of an automatic system for controlling and monitoring water supply", in 2012. 